\title{
LHX3 is an early stage and radiosensitivity prognostic biomarker in lung adenocarcinoma
}

\author{
XIN LIN $^{1^{*}}$, YAN LI $^{1 *}$, JIN WANG $^{2}$, FEI HAN $^{3}$, SHUANG LU $^{1}$, \\ YU WANG ${ }^{4}$, WENJIAN LUO ${ }^{4}$ and MINGQIAN ZHANG ${ }^{1}$ \\ ${ }^{1}$ Department of Emergency, Yan'an Hospital, Kunming Medical University, Kunming, Yunnan; \\ ${ }^{2}$ Department of Emergency, The First Affiliated Hospital of Kunming Medical University, Kunming, Yunnan; \\ ${ }^{3}$ Institute of Toxicology, College of Preventive Medicine, Third Military Medical University, Chongqing; \\ ${ }^{4}$ Department of Gerontology, Yan'an Hospital, Kunming Medical University, Kunming, Yunnan, P.R. China
}

Received February 5, 2017; Accepted July 3, 2017

DOI: $10.3892 /$ or.2017.5833

\begin{abstract}
Non-small cell lung cancer (NSCLC) is the most common type of lung cancer. We previously identified LHX3 as a new preferentially expressed gene in NSCLC. In the present study, we sought to determine its expression, the clinical relevance and the functional roles in NSCLC. LHX3 expression is sharply increased in carcinoma tissues compared to non-carcinoma tissues. Relational analysis reveals a significant association between LHX3 expression and clinical stage $(n=172, P=0.032)$ or radiotherapy $(n=167$, $\mathrm{P}=0.022$ ) of patients. LHX3 expression is much higher in the patients at advanced stages (stage III-IV) than in the patients at early stages (stage I-II, $\mathrm{P}=0.0304$ ), and LHX3 expression is remarkably increased in the patients with radiotherapy treatment $(\mathrm{P}=0.0002)$. Survival analyses indicate that LHX3 is associated with unfavorable survival $(\mathrm{n}=180, \mathrm{P}=0.002)$ and represents an independent prognostic factor [hazard ratio $(\mathrm{HR})=1.834, \mathrm{P}=0.004]$ of the NSCLC patients. Furthermore, LHX3 is associated with unfavorable overall survival $(n=866$, $\mathrm{P}=0.004)$ and represents an independent prognostic factor $(\mathrm{HR}=2.36, \mathrm{P}=0.000)$ in lung adenocarcinoma (ADC) patients, but is not associated with overall survival of squamous cell carcinoma ( $\mathrm{SCC})$ patients $(\mathrm{n}=524, \mathrm{P}=0.27)$. Further analyses found that LHX3 is an early-stage $(\mathrm{n}=94, \mathrm{P}=0.003)$ and radiosensitivity $(\mathrm{n}=45, \mathrm{P}=0.002)$ prognostic factor in $\mathrm{ADC}$ patients. The patients without radiotherapy have a significantly prolonged survival compared to those with radiotherapy $(\mathrm{P}=0.0069)$. Further functional studies show that forced expression of LHX3 in lung cancer cells obviously promotes cell proliferation and invasion, whereas inhibits cell apoptosis.
\end{abstract}

Correspondence to: Dr Mingqian Zhang, Department of Emergency, Yan'an Hospital, Kunming Medical University, No. 245 East Renming Road, Kunming, Yunnan 650051, P.R. China

E-mail: 2289462942@qq.com

*Contributed equally

Key words: LHX3, overall survival, prognostic biomarker, oncogene, non-small cell lung cancer
In summary, LHX3 is an early-stage and radiosensitivity prognostic biomarker, and a novel potential oncogene in ADC.

\section{Introduction}

Lung cancer is one of the most common cancers in the world (1). Non-small cell lung cancer (NSCLC), which mainly contains lung adenocarcinoma (ADC) and squamous cell carcinoma (SCC), is the most frequent type (accounting for $\sim 80 \%$ ) of lung cancer (2-4). The prognosis of NSCLC patients remains poor with a 5 -year survival rate being less than $15 \%(5,6)$. Therefore, searching for a useful prognostic biomarker is crucial to identify the patients with high risk of recurrence and prolong the survival of NSCLC patients.

LIM homeobox ( $\mathrm{LH})$ members are one of the most important subfamilies of LIM-homeodomain proteins. There are at least 12 LH genes in mammalian genomes. Studies have shown that these genes are critical for the development of specialized cells in various tissues (7-12). Although many reports have identified important roles on organism development, little is known about functions of these proteins in disease. Recent studies have shown that some LH genes play roles in various cancers, including $\operatorname{Lhx} 1, \operatorname{Lhx} 2$ and Lhx4 (13-18). LHX3, located at $9 \mathrm{q} 34.3$, is involved in the development of spinal cord motor neurons and pituitary (19-22). Only recently, LHX3 methylation pattern has been shown to be associated with breast cancer (23). However, the expression, prognostic significance and functional role of LHX3 remain unclear in cancer.

In the present study, we identified LHX3 as a differentially expression gene in NSCLC tumor and non-tumor tissues by RT-PCR, RT-qPCR and immunohistochemistry. Next, the prognostic significance and functional roles of LHX3 were explored. This study indicates that LHX3 is an important early-stage and radiosensitivity prognostic factor and acts as a novel oncogene in ADC.

\section{Materials and methods}

Patient samples and cell lines. A total of 180 lung adenocarcinoma patients (undergone surgical resection between 2005 and 2009) were obtained from the Yan'an Hospital in Kunming, 
China. The clinicopathological information includes sex, age, lymph node status, tumor size, histological grade, tumor location, clinical stage, radiotherapy and overall survival (OS) rates of 5-10 years. This study was approved by the ethics committee of the Yan'an Hospital Affiliated to Kunming Medical University, and all experiments were carried out in accordance with the approved guidelines of Kunming Medical University. Informed consent was signed by all the patients.

The NSCLC cell lines (A549, H460 and SPC-A1) were obtained from the Cell Bank of the Chinese Academy of Science (Shanghai, China), cultured in F12K (Sigma-Aldrich, St. Louis, MO, USA) and RPMI-1640 (HyClone Laboratories, Inc., Logan, UT, USA) supplemented with $10 \%$ fetal bovine serum (FBS; HyClone Laboratories).

Total RNA isolation. Total RNAs were extracted from frozen tissues. Approximately $3.0 \mu \mathrm{g}$ of total RNAs was treated with DNase I to eliminate genomic DNA contamination. The treated RNAs were then reverse-transcribed to cDNAs.

RT-PCR and RT-qPCR. Series of PCRs with different cycles were performed and appropriate cycles were chosen for RT-PCR. Human $\beta$-actin was used as endogenous control. The primers were: (5'-3'): LHX3-F, AAG TGT CTC AAG TGC AGC GA and LHX3-R, GTG GTA CAC GAA GTC CTG GG; $\beta$-actin-F, TTC TAC AAT GAG CTG CGT GTG and $\beta$-actin-R, GGG GTG TTG AAG GTC TCA AA. RT-qPCR was performed using the iQ5 Real-Time Detection system (Bio-Rad Laboratories, Hercules, CA, USA) and GoTaq ${ }^{\circledR}$ qPCR Master Mix (Promega, Madison, WI, USA). The expression was calculated by the equation $2^{-\Delta \Delta C T}$. The primers are $\left(5^{\prime}-3^{\prime}\right)$ : LHX3-F1, ACG GAC CCA GTT CTG ACC TA and LHX3R1, TGG TCT ACC TCA TCC AGC CA; $\beta$-actin-F2, TGA CGT GGA CAT CCG CAA AG and $\beta$-actin-R2, CTG GAA GGT GGA CAG CGA GG. All assays were performed in triplicate.

Tissue microarray (TMA). To construct the TMA slides, two cores were taken from cancerous and adjacent non-cancerous tissue (within a distance of $25 \mathrm{~mm}$ ). The adjacent noncancerous tissues were stained with hematoxylin-eosin $(\mathrm{H} \& \mathrm{E})$ and compared with normal tissues. The TMAs were then constructed as previously described (24).

Immunohistochemical analysis. IHC was performed using LHX3 antibody (1:200; Abcam) as previously described (25). Tumor cell staining was quantified and classified into 5 grades: $<10 \%$ positive cells for $0 ; 10-25 \%$ for $1 ; 26-50 \%$ for $2 ; 51-75 \%$ for 3 and $\geq 76 \%$ for 4 . Staining intensity was graded as negative (0), weak (1), moderate (2) and strong (3). The expression levels were defined by product of grade and intensity for staining.

Construction of LHX3 overexpression vector. Full-length human LHX3 was cloned, verified by sequencing and subcloned into the pIRES2-EGFP vector (Invitrogen, Carlsbad, CA, USA). Cancer cells were transfected using Lipofectamine 2000 (Invitrogen).

Western blot analysis. Protein was run on 12\% SDS-PAGE, transferred to PVDF membrane (Millipore, Billerica, MA,
USA) and incubated with primary LHX3 antibody (1:1,500; Abcam). The proteins on the membrane were detected by chemiluminescence (Pierce, Rockford, IL, USA). The same membrane was also incubated with $\beta$-actin antibody $(1: 2,000$; Sigma-Aldrich).

Cell viability assay. Transfected cells were plated in 96-well plate at 4,000 cells/well. Cell viability was evaluated by MTS (Promega) at 1-5 days as previously described (26). The experiments were carried out in triplicate.

Flow cytometry assay. Cancer cell apoptosis was determined by Annexin V and APC/7-AAD dual staining Detection kit (Nanjing KeyGen Biotech, Co., Ltd., Nanjing, China) at $48 \mathrm{~h}$ after transfection according to the instructions. The apoptosis cells were analyzed by the FACSCalibur and FlowJo software (Tree Star, Inc., San Carlos, CA, USA).

Invasion assay. Transwell assays were performed in 12-well plates with Matrigel ( $8 \mu \mathrm{m}$; Corning, Inc., Corning, NY, USA). The transfected cells were suspended in the upper well of the chamber with serum-free medium. The lower well contained media supplemented with $10 \%$ FBS. The cells of the lower chamber were stained with $0.1 \%$ crystal violet and counted. The experiments were performed in triplicate.

Clinical microarray database analysis. A Kaplan-Meier Plotter (http://kmplot.com/analysis/index.php) containing the gene expression data and survival information of lung patients was used (27). The probe was 221670_s_at. The patients were grouped according to the auto selection of best cut-off. The $\mathrm{P}<0.05$ was considered to be statistically significant.

Statistical analysis. Statistical analyses were performed using the SPSS 17.0 software (SPSS, Inc., Chicago, IL, USA). The difference in categorical variables was analyzed by the Chi-square or the Linear-by-Linear test. Kaplan-Meier method and log-rank test were used to calculate and evaluate the OS. Cox regression was used for multivariate analysis of prognostic predictors. The high or low expression was categorized by median score. The $\mathrm{P}<0.05$ was taken as statistically significant.

\section{Results}

Increased LHX3 expression is observed in NSCLC tissues. Our previous study revealed that LHX3 might be a preferentially expressed gene in NSCLC tissues. To further investigate the LHX3 expression pattern, the expression of LHX3 was determined in normal and tumor tissues by RT-PCR and RT-qPCR. The results indicated that LHX3 expression was elevated in tumor tissues compared to normal tissues (Fig. 1A). The relative mean expression level of LHX3 was 4.12 \pm 0.39 in tumor tissues remarkably higher than $1.10 \pm 0.19$ in normal tissues (Fig. 1B). To confirm the altered expression of LHX3 at protein level, we performed immunohistochemistry (IHC) for LHX3 on tissue microarray (TMA) containing 180 lung adenocarcinoma and corresponding para-carcinoma tissues. From the staining, LHX3 protein was also increased in carcinoma tissues compared to para-carcinoma tissues (Fig. 1C). The positive staining of tumor cells was then quantified 
A

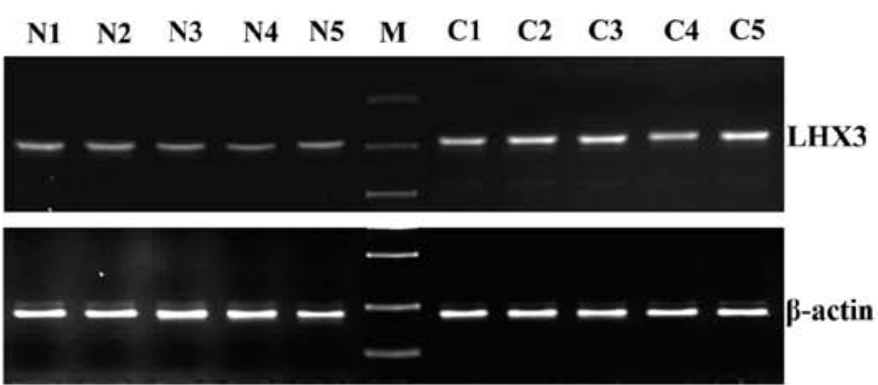

C

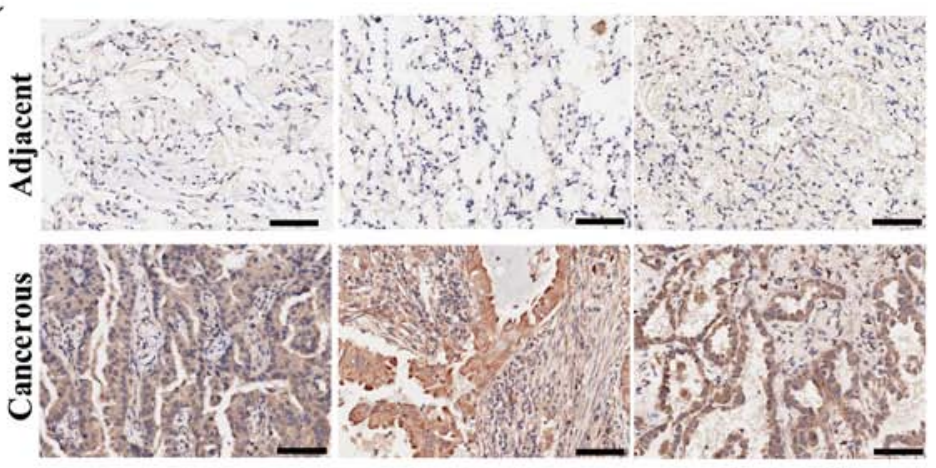

B
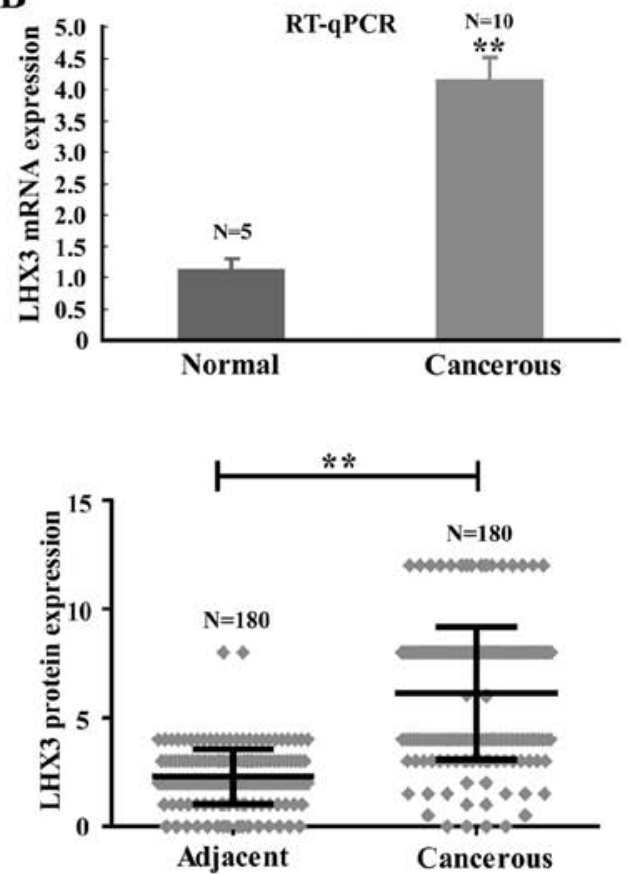

D

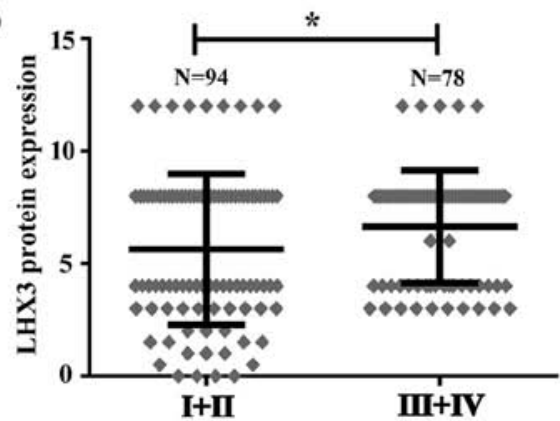

$\mathbf{E}$

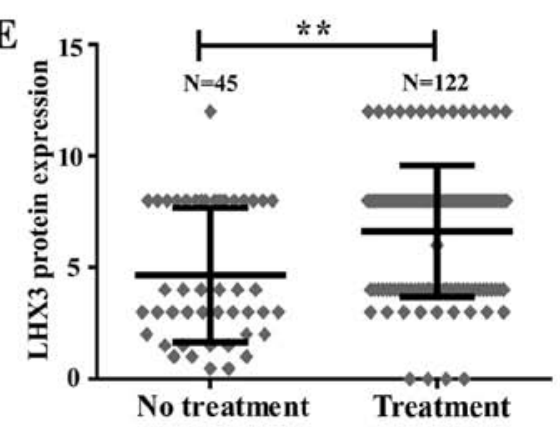

Figure 1. LHX3 expression is increased in cancerous tissue. (A) RT-PCR analysis of LHX3 mRNA expression in normal and cancerous tissues. N, normal lung tissues; M, marker; C, cancerous tissues. The $\beta$-actin was used as internal control. (B) Analysis of LHX3 mRNA levels in normal and cancerous tissues by qRT-PCR. Five different normal lung and ten different cancerous lung tissues were mixed together. $\beta$-actin was used as internal control. ${ }^{* *} \mathrm{P}<0.01$. Error bar indicates SD; $(n=3)$. (C) Immunohistochemical analysis of LHX3 protein expression in 180 cancerous and adjacent non-cancerous samples. Scale bars represent $50 \mu \mathrm{m}$. LHX3 expression was higher in cancerous tissues than adjacent non-cancerous tissues. ${ }^{* *} \mathrm{P}<0.01$. (D and E) LHX3 protein expressions are shown in the patients at different clinical stages or radiotherapy treatments. ${ }^{*} \mathrm{P}<0.05 .{ }^{* *} \mathrm{P}<0.01$.

using the scoring system for intensity and positive staining percentage. The mean protein expression level of LHX3 was $6.13 \pm 3.06$ in cancer tissues sharply higher than $2.29 \pm 1.27$ in para-carcinoma tissues $(\mathrm{P}<0.0001$; Fig. $1 \mathrm{C})$. In addition, LHX3 was expressed in both the nucleus and cytoplasm of tumor cells. These results revealed that LHX3 expression is increased in tumor tissues.

LHX3 is associated with clinical stage and radiotherapy of NSCLC patients. Based on the quantified expression above, we classified LHX3 expression into high and low groups (the scores greater than or less than the mean expression were obtained in two groups: low and high). After investigating association between LHX3 expression and clinicopathological features, LHX3 expression was found to be associated with clinical stage $(n=172, P=0.032)$ and radiotherapy $(n=167$, $\mathrm{P}=0.022$ ) of the NSCLC patients (Table I). However, LHX3 expression was not associated with age $(\mathrm{P}=0.359)$, histological grade $(\mathrm{P}=0.865)$, sex $(\mathrm{P}=0.550)$, tumor size $(\mathrm{P}=0.368)$, tumor location $(\mathrm{P}=0.171)$ or lymph node status $(\mathrm{P}=0.361)$ of the patients (Table I). To further confirm the association between LHX3 expression and clinical stage or radiotherapy, we then analyzed the changes of LHX3 expression in the patients at early stages (stage I-II, $\mathrm{n}=94$ ) or advanced stages (stage III-IV, $\mathrm{n}=78)$ and the patients with $(\mathrm{n}=122)$ or without $(\mathrm{n}=45)$ radiotherapy treatment. The data indicated that LHX3 expression is much higher in the patients at advanced stages than in the patients at early stages ( $\mathrm{P}=0.0304$; Fig. 1D) and LHX3 expression is clearly increased in the patients with radiotherapy treatment $(\mathrm{P}=0.0002$; Fig. 1E).

LHX3 expression is associated with poor OS of NSCLC patients. LHX3 expression was then analyzed with respect to survival data of the patients. Kaplan-Meier analysis revealed a poor OS in the patients with high LHX3 expression compared to that with low LHX3 expression ( $\mathrm{P}=0.002$; Fig. $2 \mathrm{~A})$. To correct for bias, LHX3 expression as well as other parameters (sex, age, histological grade, tumor size, tumor location, lymph 

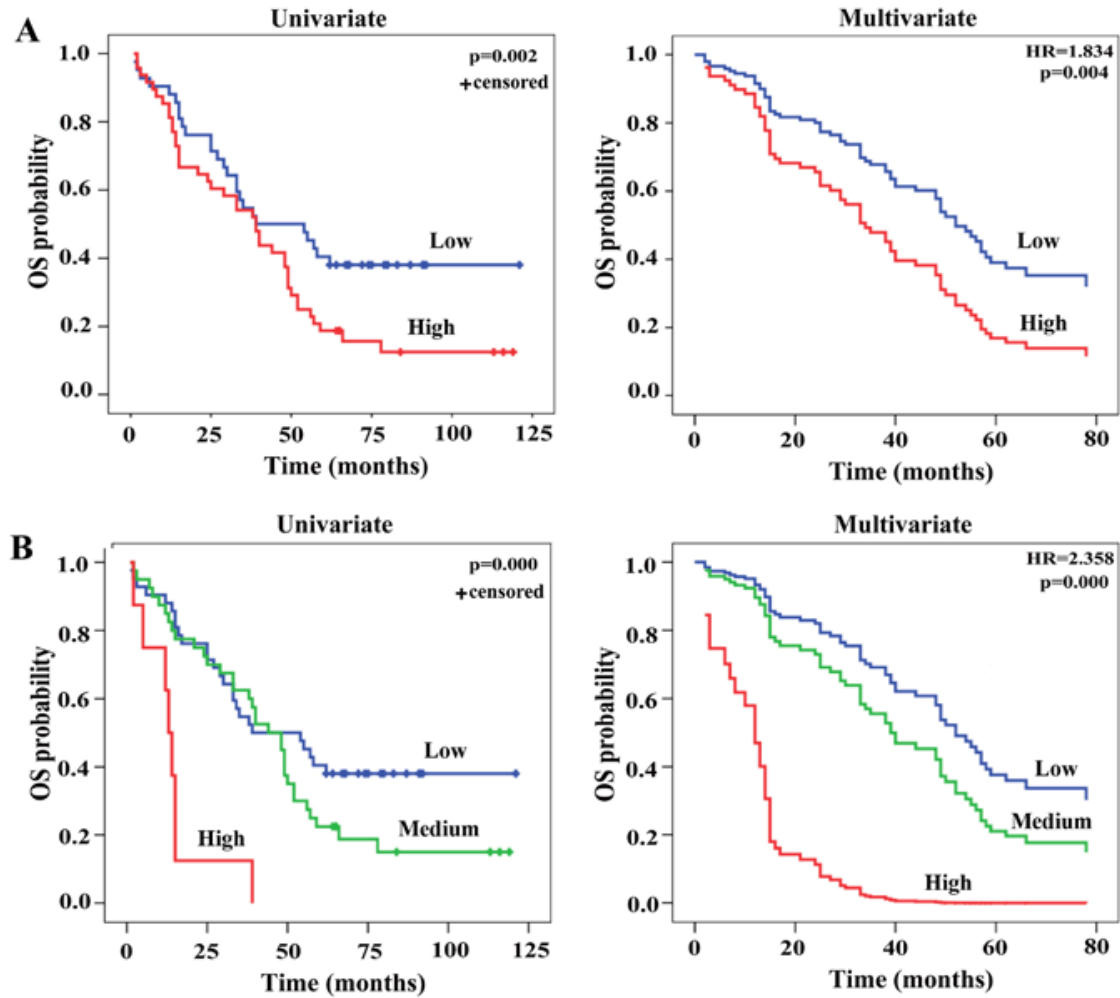

Figure 2. LHX3 expression is associated with poor OS of NSCLC patients. (A) Kaplan-Meier and Cox regression survival analysis of LHX3 expression in 180 NSCLC patients divided into two groups. LHX3 is associated with poor OS of the patients, and is an independent prognostic factor. HR, hazard ratio; low, staining weak and moderate; high, staining strong. (B) Kaplan-Meier and Cox regression survival analysis of LHX3 expression in 180 NSCLC patients divided into three groups. LHX3 is also associated with poor OS and is an independent prognostic factor. Low, staining weak; medium, staining moderate; high, staining strong.
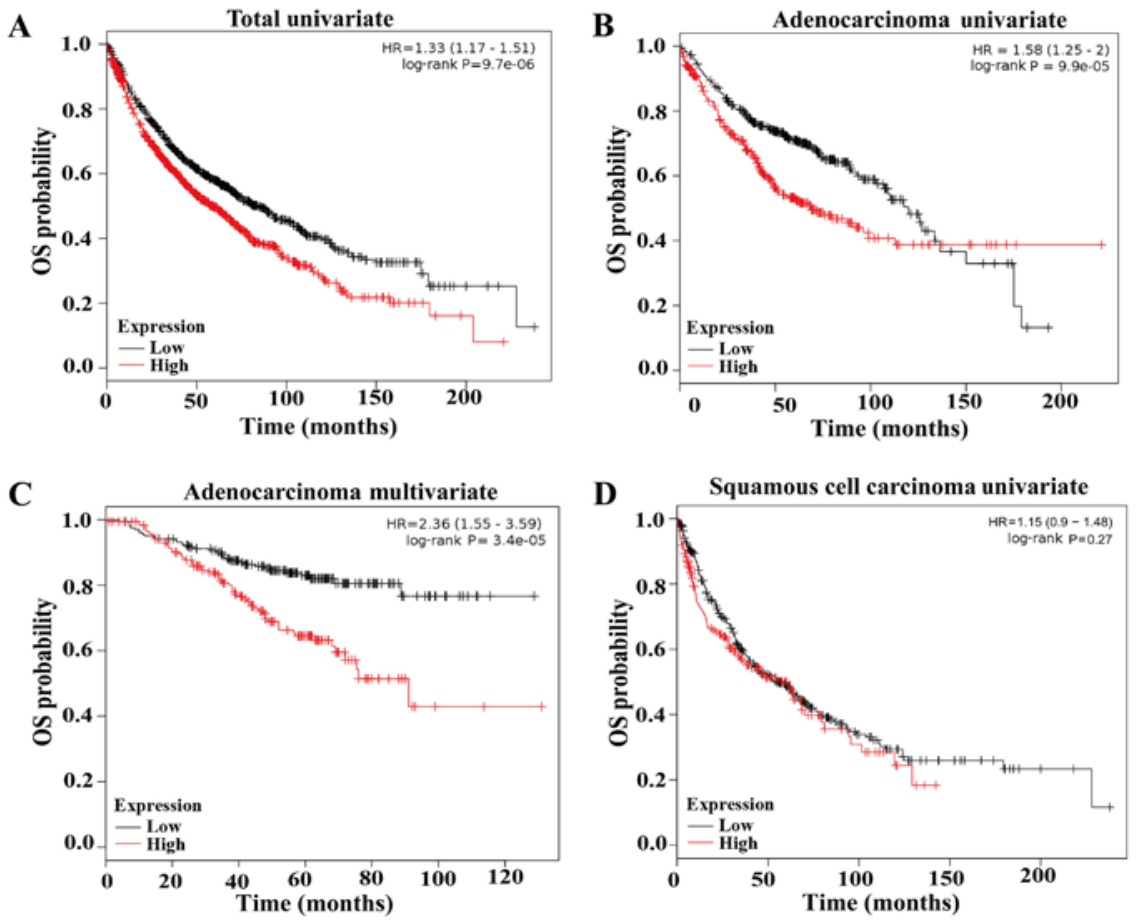

Figure 3. LHX3 is associated with poor OS of ADC but not of SCC patients. (A) Kaplan-Meir survival analysis between LHX3 expression and OS of 1928 lung cancer patients in Kaplan-Meier Plotter (http://kmplot.com/analysis/index). The OS of LHX3 high expression patients was shorter than that of LHX3 low expression patients. Auto best cut-off was chosen; cut-off value was 64 and the expression range was 2-914. (B) Kaplan-Meir analysis between LHX3 expression and OS of 866 ADC patients in Kaplan-Meier Plotter. The OS of the patients with high LHX3 was shorter than that of the patients with low LHX3. Auto best cut-off was chosen in the analysis; cut-off value was 44 and the expression range was 3-774. (C) Multivariate Cox regression analysis of LHX3 expression with OS of 387 ADC patients in Kaplan-Meier Plotter. Auto select best cut-off was chosen in the analysis; cut-off used was 41 and the expression range was 5-751. (D) Kaplan-Meir analysis between LHX3 expression and OS of 524 SCC patients in Kaplan-Meier Plotter. Auto best cut-off was chosen in the analysis; cut-off value was 241 and the expression range was 2-705. 
Table I. Correlations of LHX3 with clinicopathological features of patients $(n=180)$.

\begin{tabular}{|c|c|c|c|c|}
\hline \multirow[b]{2}{*}{ Clinical features } & \multirow[b]{2}{*}{ Total } & \multicolumn{2}{|c|}{ LHX3 expression } & \multirow[b]{2}{*}{ P-value } \\
\hline & & $\begin{array}{l}\text { High } \\
(\mathrm{n}=96)\end{array}$ & $\begin{array}{c}\text { Low } \\
(\mathrm{n}=84)\end{array}$ & \\
\hline Age (years) & & & & 0.359 \\
\hline$<60$ & 70 & 34 & 36 & \\
\hline$\geq 60$ & 110 & 62 & 48 & \\
\hline Histological grade & & & & 0.865 \\
\hline 1 & 22 & 12 & 10 & \\
\hline 2 & 116 & 62 & 54 & \\
\hline 3 & 42 & 22 & 20 & \\
\hline Sex & & & & 0.550 \\
\hline Male & 98 & 50 & 48 & \\
\hline Female & 82 & 46 & 36 & \\
\hline Clinical stage & & & & 0.032 \\
\hline $\mathrm{I}+\mathrm{II}$ & 92 & 42 & 50 & \\
\hline $\mathrm{III}+\mathrm{IV}$ & 80 & 50 & 30 & \\
\hline Tumor size $(\mathrm{cm})$ & & & & 0.368 \\
\hline$<4$ & 80 & 46 & 34 & \\
\hline$\geq 4$ & 100 & 50 & 50 & \\
\hline Tumor location & & & & 0.171 \\
\hline Right & 102 & 60 & 42 & \\
\hline Left & 76 & 36 & 40 & \\
\hline Lymph node status & & & & 0.361 \\
\hline Negative & 78 & 38 & 40 & \\
\hline Positive & 96 & 54 & 42 & \\
\hline Radiotherapy & & & & 0.022 \\
\hline Yes & 122 & 72 & 50 & \\
\hline No & 45 & 17 & 28 & \\
\hline
\end{tabular}

node, clinical stage and radiotherapy) were analyzed by multivariate Cox-regression analysis. In addition to clinical stage [hazard ratio $(\mathrm{HR})=1.545, \mathrm{P}=0.001$ ], $\mathrm{LHX} 3$ expression is an independent prognostic factor (HR, 1.834, $\mathrm{P}=0.004)$ for $\mathrm{OS}$ of the patients (Fig. 2A and Table II). To confirm these results, the survival analyses were also performed by Kaplan-Meier and Cox-regression methods base on three groups of LHX3 expression: low, intermediate and high. The results observed were consistent with the ones based on two groups (HR, 2.358, $\mathrm{P}=0.000$; Fig. 2B). To further validate the correlation between the LHX3 expression and the survival of patients, the contribution of LHX3 to survival of patients was also examined in a clinical microarray database (http://kmplot.com/analysis/ index, 27). The analyses revealed that LHX3 expression still predicts poor OS of patients (Fig. 3A). These findings show that LHX3 is an unfavorable and independent prognostic factor of NSCLC patients.

LHX3 expression is associated with poor OS outcomes in $A D C$ patients. We then investigated the correlations between the LHX3 expression and the survival of ADC and SCC patients, respectively. In ADC patients, survival analysis indicated that the patients with low LHX3 expression had a significantly prolonged OS compared to those with high LHX3 expression $(\mathrm{HR}=2.36$, multivariate $\mathrm{P}=0.000$ /univariate $\mathrm{P}=0.000$; Fig. $3 \mathrm{~B}$ and $\mathrm{C}$ ). In SCC patients, survival analysis demonstrated that LHX3 expression was not associated with OS of the patients $(\mathrm{P}=0.27$; Fig. 3D). These results reveal that LHX3 is an unfavorable and independent prognostic factor of ADC patients, but is not associated with OS of SCC patients.

LHX3 is an early-stage prognostic factor of ADC patients. The associations between LHX3 expression and survival of ADC patients at early stages (stage I-II, $\mathrm{n}=94$ ) or advanced stages (stage III-IV, n=78) were further evaluated. Kaplan-Meier and Cox-regression analyses indicated that LHX3 expression was obviously associated with the OS $(\mathrm{P}=0.003)$ and represented an independent prognostic factor of ADC patients at early stage (HR, 2.059, $\mathrm{P}=0.045$; Fig. 4A), but not associated with $\mathrm{OS}$ of the ones at advanced stage ( $\mathrm{P}=0.389$; Fig. 4B). These data indicate that LHX3 is an early stage prognostic biomarker of ADC patients.

LHX3 is a radiosensitivity prognostic biomarker of $A D C$ patients. We then ascertained whether LHX3 expression predicted poor OS of ADC patients treated with or without radiotherapy. From the results, we found a significant effect of LHX3 expression on poor OS of the patients without radiotherapy treatment ( $\mathrm{P}=0.009$; Fig. $4 \mathrm{C})$, while LHX3 expression was not associated with OS of the patients with radiotherapy treatment $(\mathrm{P}=0.209$; Fig. 4D). To further validate whether radiotherapy treatment has an effect on ADC patient survival, we analyzed the survival time of the patient treated with or without radiotherapy. The results showed that the patient without radiotherapy (54.2 months) had a significantly prolonged survival compared to those with radiotherapy (40.15 months, $\mathrm{P}=0.0069$; Fig. 4E). These results demonstrate that LHX3 is a radiosensitivity prognostic biomarker of $\mathrm{ADC}$ patients.

LHX3 promotes cancer cell growth and proliferation. To evaluate the possible roles of LHX3, we examined cancer cell growth and proliferation by MTS assay with exogenous expression of LHX3 in A549 (human adenocarcinoma cell line), H460 (the human large cell lung cancer cell line) and SPC-A1 (human adenocarcinoma cell line) cells. The results of MTS assay indicated that ectopic expression of LHX3 significantly promoted cancer cell growth and proliferation compared with the empty vector control (Fig. 5). These data indicate that LHX3 remarkably activates cancer cell proliferation and growth.

LHX3 inhibits cancer cell apoptosis. To determine whether the promotion of cancer cell proliferation of LHX3 related to cell apoptosis, we conducted Annexin V-APC/7AAD (aminoactinomycin $\mathrm{D}$ ) double staining the transfected cancer cells followed by flow cytometric analysis. The experimental data showed that LHX3 overexpression resulted in a significant decrease of early apoptotic cells and late apoptotic cells in A549 and H460 (Fig. 6). These results demonstrate that LHX3 markedly inhibits cancer cell apoptosis. 
Table II. Multivariate analysis of prognostic factors in NSCLC patients $(n=180)$.

\begin{tabular}{|c|c|c|c|c|}
\hline Expression & Variables & HR & $95 \% \mathrm{CI}$ & P-value \\
\hline \multirow[t]{9}{*}{ The LHX3 expression } & LHX3 expression & 1.834 & $1.209-2.780$ & 0.004 \\
\hline & Gender & 1.352 & $0.893-2.048$ & 0.154 \\
\hline & Age & 1.001 & $0.979-1.023$ & 0.930 \\
\hline & Histological grade & 1.101 & $0.781-1.554$ & 0.582 \\
\hline & Tumor size & 1.090 & $0.980-1.212$ & 0.110 \\
\hline & Tumor location & 1.122 & $0.738-1.705$ & 0.591 \\
\hline & Lymph node status & 1.030 & $0.985-1.078$ & 0.190 \\
\hline & Clinical stage & 1.545 & $1.195-1.996$ & 0.001 \\
\hline & Radiotherapy & 1.454 & $0.906-2.334$ & 0.121 \\
\hline
\end{tabular}

HR, hazard ratio; CI, confidence interval. Bold, statistically significant.
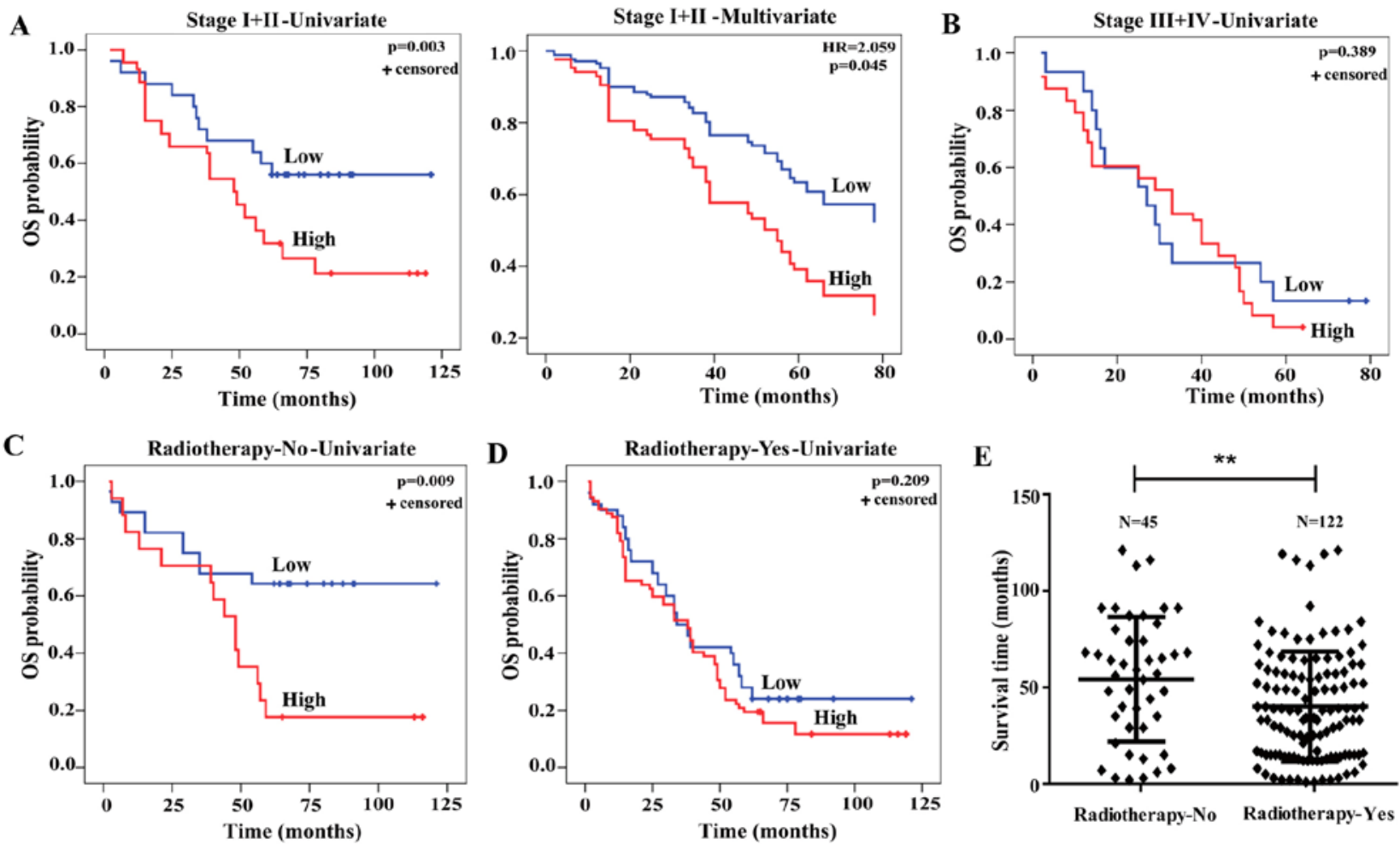

Figure 4. LHX3 is an early stage and radiosensitivity prognostic factor of the ADC patients. (A) Kaplan-Meier and Cox regression survival analysis of LHX3 expression in 94 early stage (stage I-II) ADC patients. LHX3 is associated with poor OS of the patients, and is an independent prognostic factor. HR, hazard ratio. (B) Kaplan-Meier survival analysis of LHX3 expression in 78 advanced stage (stage III-IV) ADC patients. (C and D) Kaplan-Meier survival analyses of LHX3 expression in 45 patients without radiotherapy or 122 patients with radiotherapy. (E) The survival time of the patients without radiotherapy are shown compared to that of the patients with radiotherapy. ${ }^{* *} \mathrm{P}<0.01$.

LHX3 stimulates cancer cell invasion. To investigate the effects of LHX3 on cancer cell invasion, Transwell assays with Matrigel were performed. A significant increase of cancer cell invasion was found in LHX3-transfected cells compared with empty vector-transfected cells (Fig. 7). These data suggest that LHX3 markedly stimulates cancer cell invasion.

\section{Discussion}

In the present study, the expression, the clinical relevance and the functional roles of LHX3 were investigated in NSCLC for the first time. Our data reveal that LHX3 is generally increased in cancer tissues; LHX3 is obviously associated with clinical stage and radiotherapy of NSCLC patients, and is an unfavorable independent prognostic factor of ADC patients; furthermore, LHX3 is an early stage and radiosensitivity prognostic factor in ADC patients; functionally, LHX3 promotes cancer cell proliferation and invasion, whereas inhibits cancer cell apoptosis.

Previous studies have shown that some LH genes are involved in various cancers progression (13-18). However, little is known about their clinical relevance. In the present study, to 
A
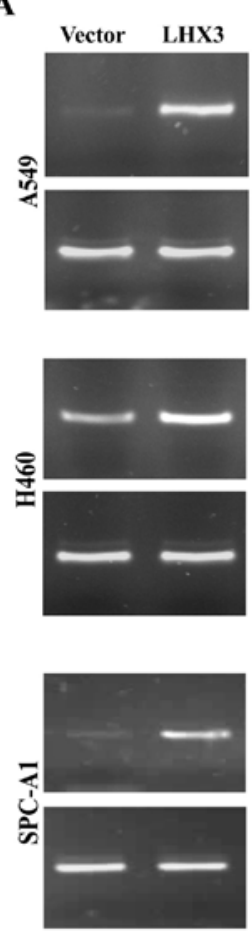
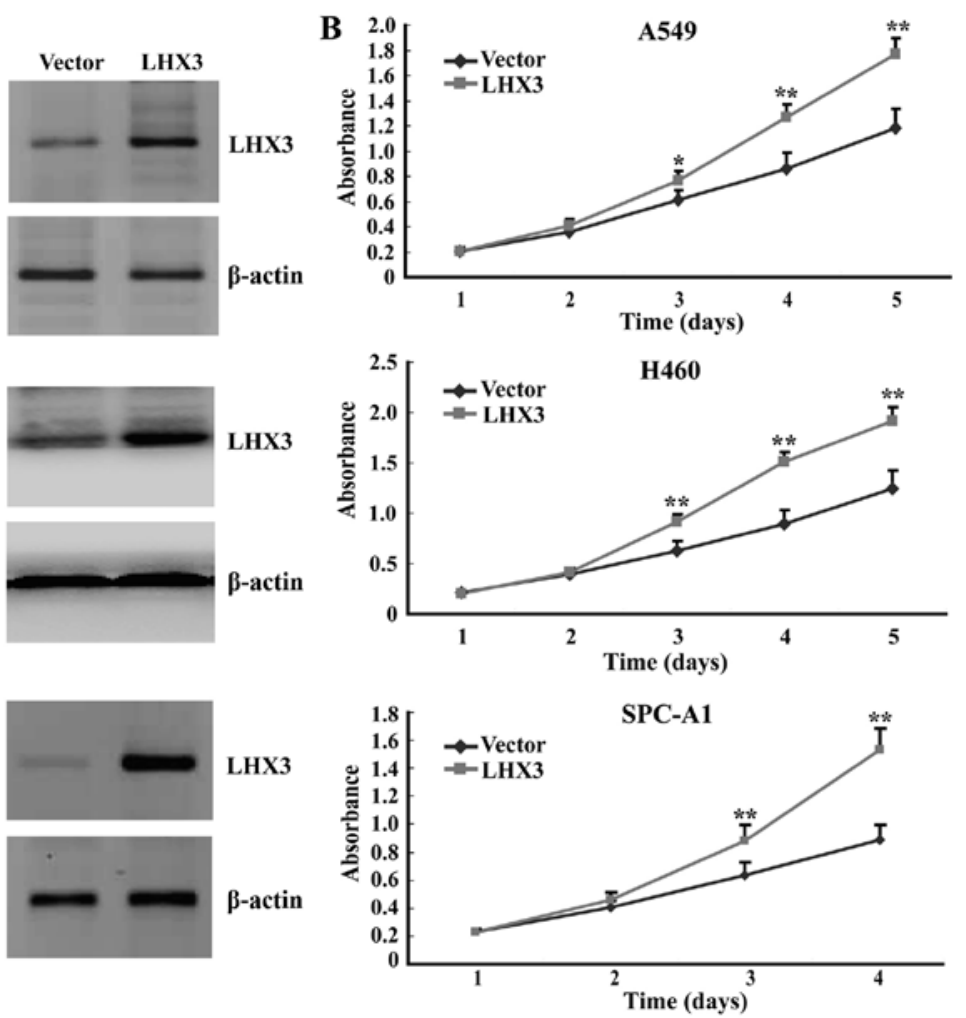

Figure 5. LHX3 promotes cancer cell growth and proliferation. (A) Transfectants of LHX3 or empty vector plasmids were identified by RT-PCR and western blot analysis in A549, H460 and SPC-A1. Strong LHX3 expression was detected after LHX3 plasmid transfection, but not after empty vector plasmid transfection. (B) MTS assays were performed to examine the effect of LHX3 on cancer cell growth and proliferation. Cell proliferation was evaluated by CellTiter 96 AQueous One Solution Cell Proliferation assay (Promega) in triplicate. ${ }^{* *} \mathrm{P}<0.01$.

A

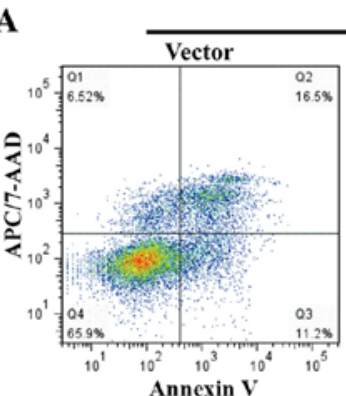

A549
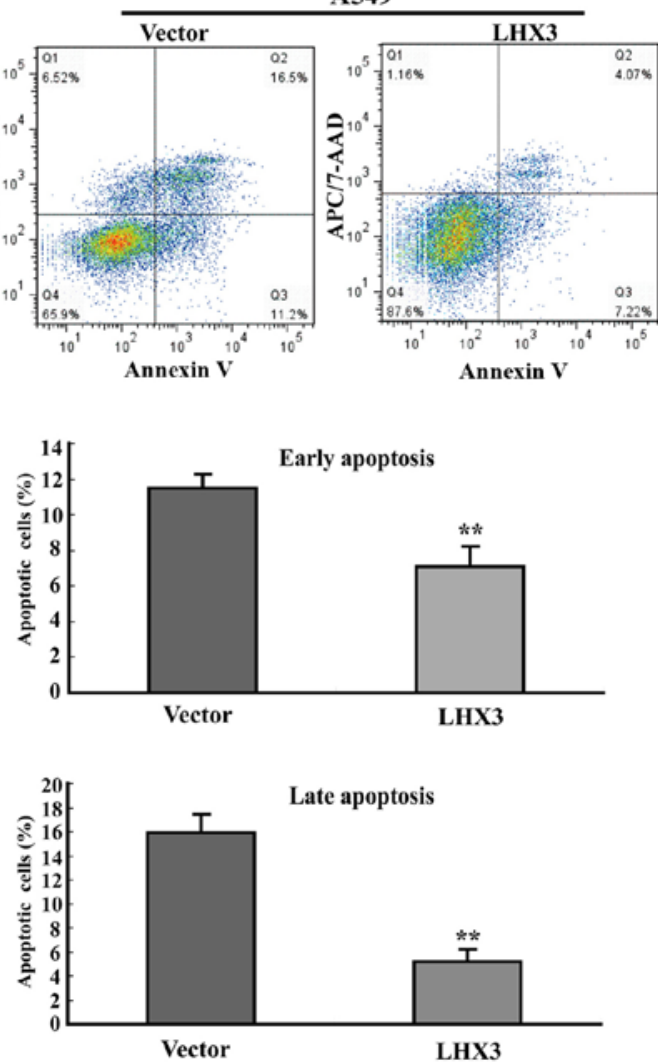

B
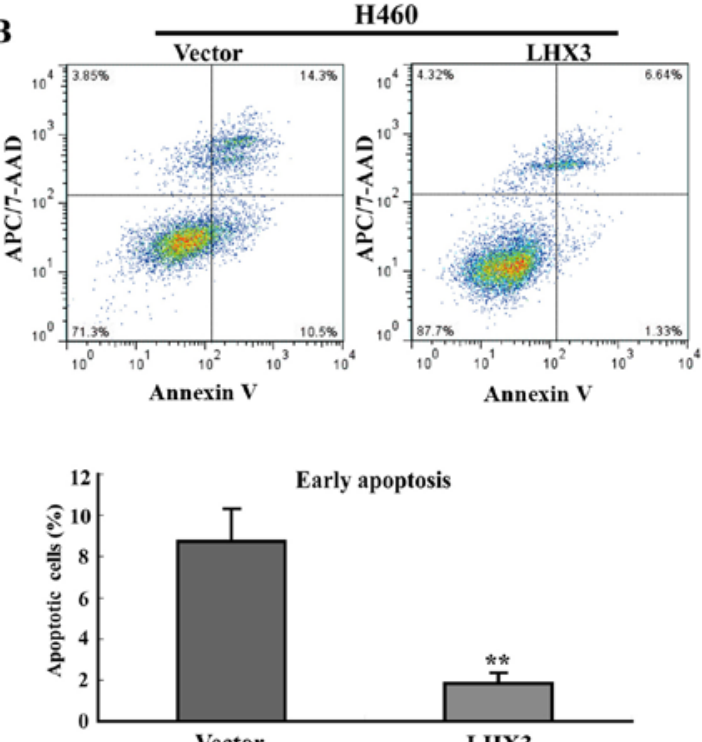

Vector

LHX3

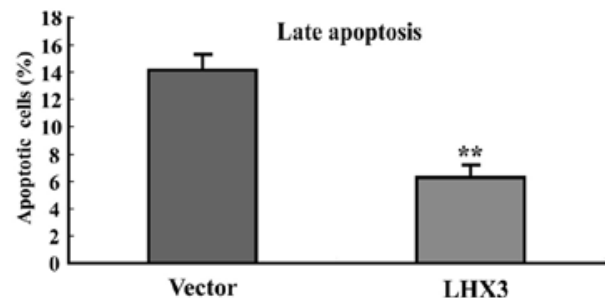

Figure 6. LHX3 inhibits cancer cell apoptosis. (A) Flow cytometry assay with double staining was performed to test cancer cell apoptosis after transfecting LHX3 or empty vector plasmids in A549. (B) Cancer cell apoptosis was evaluated by flow cytometry after transfecting LHX3 or empty vector plasmids in H460. Cell apoptosis was detected by Annexin V and APC/7-AAD staining (Nanjing KeyGen Biotech). Error bars indicate SD; (n=3). 
A

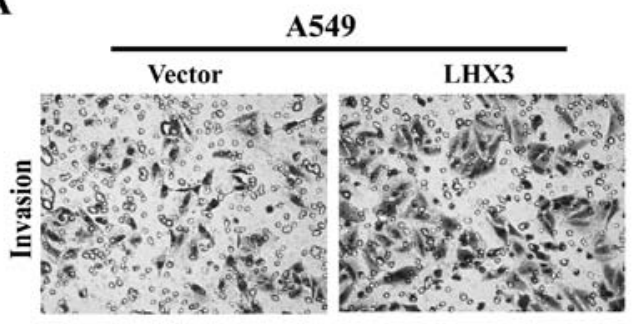

$\mathbf{B}$

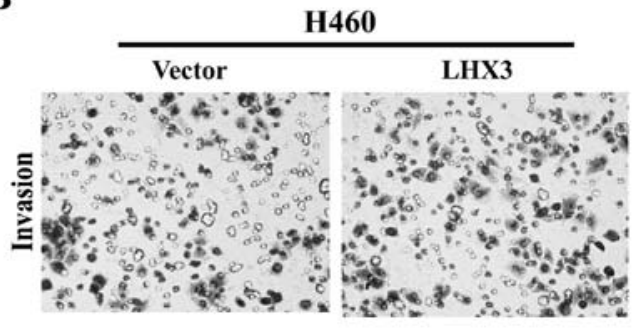

C

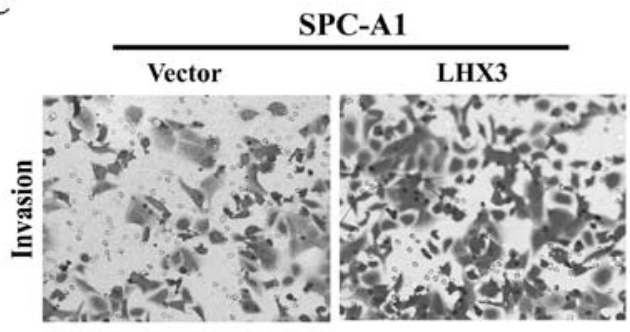

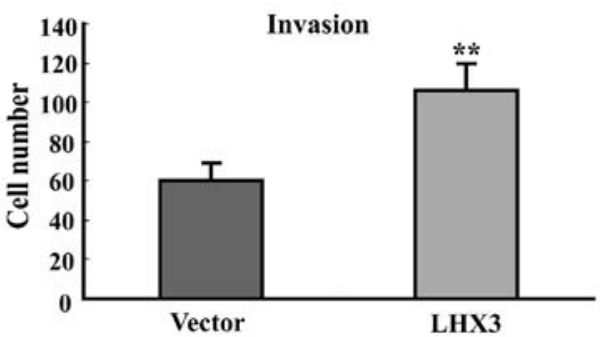
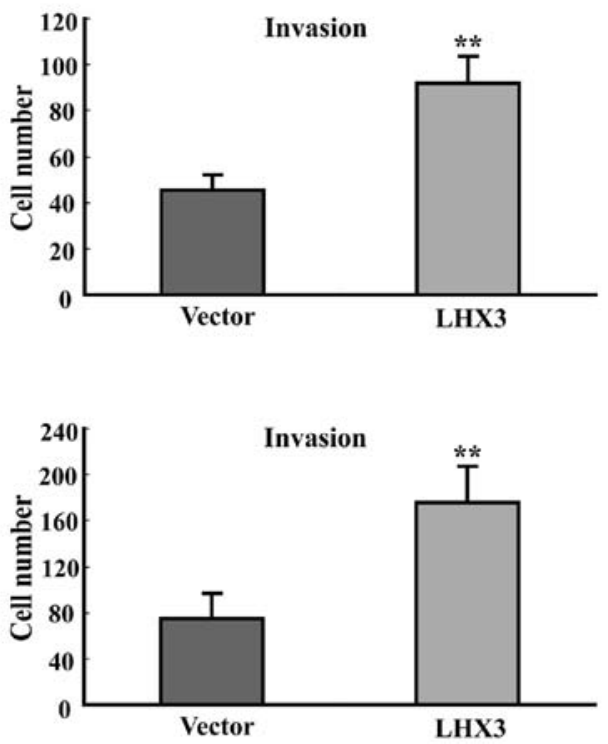

Figure 7. LHX3 stimulates cancer cell invasion. (A) The cancer cell invasion was measured by Transwell assays with Matrigel after LHX3 or empty plasmid transfection in A549. The cells in lower chamber were fixed, stained and quantified by counting. The quantified cells were determined by the average count of at least five random microscopic fields. Error bars indicate SD; $(n=5)$. (B) The cancer cell invasion was measured by Transwell assays after LHX3 or empty plasmid transfection in H460. (C) The cancer cell invasion was measured by Transwell assays after LHX3 or empty plasmid transfection in SPC-A1.

determine the clinical relevance of LHX3, relational analysis and survival analysis were performed. Relational analysis indicates that LHX3 is obviously associated with clinical stage and radiotherapy of patients. To confirm the association between the LHX3 expression and the clinical stage or radiotherapy of patients, the expression patterns were performed. LHX3 expression is higher in the advanced stage patients than that in the early stages patients, and similarly LHX3 expression is remarkably increased in the patients with radiotherapy. These results suggest a close association between LHX3 and tumor metastasis or radiotherapy treatment.

To further evaluate the prognostic significance of LHX3, Kaplan-Meier and Cox-regression analysis were performed. The patients with low LHX3 expression have prolonged OS compared to the ones with high LHX3 expression, and LHX3 is an independent prognostic factor for OS in NSCLC patients. Notably, LHX3 is an unfavorable and independent prognostic factor in ADC patients, but is not associated with survival of SCC patients. Noticeably, LHX3 is an early stage and radiosensitivity prognostic factor in ADC patients and the radiotherapy treatment significantly shorten the survival of ADC patients suggesting radiotherapy is an unfavorable therapeutic strategy for ADC patients. Considering the effect of radiotherapy on LHX3 expression, we speculate that radiotherapy as an unfavorable factor for patient survival may be mediated by upregulation of LHX3 expression.
Previous evidence has indicated that LHX3 plays an important role in spinal cord motor neurons and pituitary development (19-22). In a recent study, the DNA methylation of LHX3 has been reported to be associated with breast cancer (23). However, no studies on its function in cancer were reported until now. In the present study, LHX3 expression was evaluated in 180 NSCLC patients, and increased LHX3 expression was found in tumor tissues compared to non-tumor tissues. This result suggests that LHX3 may play roles in tumorigenesis of NSCLC. To determine the roles of LHX3, gain-of-function assays were investigated. The functional analysis show that LHX3 significantly promotes cancer cell proliferation and invasion, whereas inhibits cancer cell apoptosis. These findings indicate that LHX3 functions as a potential oncogene in cancer.

Previous studies have reported that several other LHX genes, such as LHX4, LHX6 and LHX9, are implicated in tumorigenesis, and act as tumor suppressors in different types of cancers $(16,18,28,29)$. LHX3 belongs to the same LHX family, and it is plausible to assume that LHX3 may function in a similar way. However, LHX3 was found as an oncogene in the present study. After systematic retrieval of literature, genes of the same family with different (tumor suppressive or oncogenic) roles are a common phenomenon in cancer development, such as the well known SOX and HOX family gene (30-32). We then used The UCSC Cancer Genomics Browser (https:// 
genome-cancer.soe.ucsc.edu/proj/site/hg Heatmap/) to analyze the gene expression in cancer and normal lung tissues. The results showed that decreased LHX6 expression was observed in lung cancer tissues, while increased LHX3 expression was observed in lung cancer tissues compare with normal lung tissues in the same cohort consistent with our results (data not shown). According to these data, the conclusion that LHX6 is a tumor suppressor whereas LHX3 is an oncogene in lung cancer is reasonable. Furthermore, we then evaluated the functional role of LHX3 in another lung cancer cell line (SPC-A1), and the results revealed that LHX3 also promotes cancer cell proliferation and invasion, which is consistent with the results of A549 and H460 cells. All the data suggest that LHX3 has an oncogenic role in lung cancer.

In conclusion, LHX3 is a potential early stage and radiosensitivity biomarker for prognosis evaluation of patient and acts as a candidate oncogene in ADCs. However, further researches are needed to explore the molecular mechanism of LHX3 in cancer.

\section{Acknowledgements}

The present study was supported by the National Natural Science Foundation of China (no. 81502551). The authors thank all the patients involved in this study.

\section{References}

1. Siegel R, Naishadham D and Jemal A: Cancer statistics, 2012. CA Cancer J Clin 62: 10-29, 2012.

2. Boyle P and Ferlay J: Cancer incidence and mortality in Europe, 2004. Ann Oncol 16: 481-488, 2005

3. Ridge CA, McErlean AM and Ginsberg MS: Epidemiology of lung cancer. Semin Intervent Radiol 30: 93-98, 2013.

4. Pao W and Chmielecki J: Rational, biologically based treatment of EGFR-mutant non-small-cell lung cancer. Nat Rev Cancer 10: 760-774, 2010.

5. Molina JR, Yang P, Cassivi SD, Schild SE and Adjei AA: Non-small cell lung cancer: Epidemiology, risk factors, treatment, and survivorship. Mayo Clin Proc 83: 584-594, 2008.

6. Jemal A, Siegel R, Xu J and Ward E: Cancer statistics, 2010. CA Cancer J Clin 60: 277-300, 2010.

7. Chen YT, Kobayashi A, Kwan KM, Johnson RL and Behringer RR: Gene expression profiles in developing nephrons using Lim1 metanephric mesenchyme-specific conditional mutant mice. BMC Nephrol 7: 1, 2006.

8. Shawlot W and Behringer RR: Requirement for Lim1 in headorganizer function. Nature 374: 425-430, 1995.

9. Richter K, Pinto do OP, Hägglund AC, Wahlin A and Carlsson L: Lhx 2 expression in hematopoietic progenitor/stem cells in vivo causes a chronic myeloproliferative disorder and altered globin expression. Haematologica 88: 1336-1347, 2003.

10. Raetzman LT, Ward R and Camper SA: Lhx4 and Prop1 are required for cell survival and expansion of the pituitary primordia. Development 129: 4229-4239, 2002.

11. Zhao Y, Sheng HZ, Amini R, Grinberg A, Lee E, Huang S, Taira $\mathrm{M}$ and Westphal $\mathrm{H}$ : Control of hippocampal morphogenesis and neuronal differentiation by the LIM homeobox gene Lhx5. Science 284: 1155-1158, 1999.

12. Grigoriou M, Tucker AS, Sharpe PT and Pachnis V: Expression and regulation of Lhx6 and Lhx7, a novel subfamily of LIM homeodomain encoding genes, suggests a role in mammalian head development. Development 125: 2063-2074, 1998.

13. Dormoy V, Béraud C, Lindner V, Thomas L, Coquard C, Barthelmebs M, Jacqmin D, Lang H and Massfelder T: LIM-class homeobox gene Lim1, a novel oncogene in human renal cell carcinoma. Oncogene 30: 1753-1763, 2011.
14. Gorantla B, Asuthkar S, Rao JS, Patel J and Gondi CS: Suppression of the uPAR-uPA system retards angiogenesis, invasion, and in vivo tumor development in pancreatic cancer cells. Mol Cancer Res 9: 377-389, 2011.

15. Kim MS, Lee J, Oh T, Moon Y, Chang E, Seo KS, Hoehn BD, An S and Lee JH: Genome-wide identification of OTP gene as a novel methylation marker of breast cancer. Oncol Rep 27: 1681-1688, 2012.

16. Hung TM, Hu RH, Ho CM, Chiu YL, Lee JL, Jeng YM, Shih DT and Lee PH: Downregulation of alpha-fetoprotein expression by LHX4: A critical role in hepatocarcinogenesis. Carcinogenesis 32: 1815-1823, 2011.

17. Estécio MR, Youssef EM, Rahal P, Fukuyama EE, Góis-Filho JF, Maniglia JV, Goloni-Bertollo EM, Issa JP and Tajara EH: LHX6 is a sensitive methylation marker in head and neck carcinomas. Oncogene 25: 5018-5026, 2006.

18. Jung S, Jeong D, Kim J, Yi L, Koo K, Lee J, Kim CJ, Kim CH, An S, Yang Y, et al: Epigenetic regulation of the potential tumor suppressor gene, hLHX6.1, in human cervical cancer. Int J Oncol 38: 859-869, 2011.

19. Sharma K, Sheng HZ, Lettieri K, Li H, Karavanov A, Potter S, Westphal $\mathrm{H}$ and Pfaff SL: LIM homeodomain factors Lhx3 and Lhx4 assign subtype identities for motor neurons. Cell 95: 817-828, 1998.

20. Sheng HZ, Zhadanov AB, Mosinger B Jr, Fujii T, Bertuzzi S, Grinberg A, Lee EJ, Huang SP, Mahon KA and Westphal H: Specification of pituitary cell lineages by the LIM homeobox gene Lhx3. Science 272: 1004-1007, 1996.

21. Sheng HZ, Moriyama K, Yamashita T, Li H, Potter SS, Mahon KA and Westphal H: Multistep control of pituitary organogenesis. Science 278: 1809-1812, 1997.

22. Thaler JP, Lee SK, Jurata LW, Gill GN and Pfaff SL: LIM factor Lhx 3 contributes to the specification of motor neuron and interneuron identity through cell-type-specific protein-protein interactions. Cell 110: 237-249, 2002.

23. Dietrich D, Lesche R, Tetzner R, Krispin M, Dietrich J, Haedicke W, Schuster M and Kristiansen G: Analysis of DNA methylation of multiple genes in microdissected cells from formalin-fixed and paraffin-embedded tissues. J Histochem Cytochem 57: 477-489, 2009.

24. Gao Q, Qiu SJ, Fan J, Zhou J, Wang XY, Xiao YS, Xu Y, Li YW and Tang ZY: Intratumoral balance of regulatory and cytotoxic $\mathrm{T}$ cells is associated with prognosis of hepatocellular carcinoma after resection. J Clin Oncol 25: 2586-2593, 2007.

25. Han F, Dong Y, Liu W, Ma X, Shi R, Chen H, Cui Z, Ao L, Zhang $\mathrm{H}$, Cao J, et al: Epigenetic regulation of sox 30 is associated with testis development in mice. PLoS One 9: e97203, 2014.

26. Han F, Liu W, Jiang X, Shi X, Yin L, Ao L, Cui Z, Li Y, Huang C, Cao J, et al: SOX30, a novel epigenetic silenced tumor suppressor, promotes tumor cell apoptosis by transcriptional activating p53 in lung cancer. Oncogene 34: 4391-4402, 2015.

27. Győrffy B, Surowiak P, Budczies J and Lánczky A: Online survival analysis software to assess the prognostic value of biomarkers using transcriptomic data in non-small-cell lung cancer. PLoS One 8: e82241, 2013.

28. Liu WB, Jiang X, Han F, Li YH, Chen HQ, Liu Y, Cao J and Liu JY: LHX6 acts as a novel potential tumour suppressor with epigenetic inactivation in lung cancer. Cell Death Dis 4: e882, 2013.

29. Vladimirova V, Mikeska T, Waha A, Soerensen N, Xu J, Reynolds PC and Pietsch T: Aberrant methylation and reduced expression of LHX9 in malignant gliomas of childhood. Neoplasia 11: 700-711, 2009

30. Thu KL, Becker-Santos DD, Radulovich N, Pikor LA, Lam WL and Tsao MS: SOX15 and other SOX family members are important mediators of tumorigenesis in multiple cancer types. Oncoscience 1: 326-335, 2014.

31. Thu KL, Radulovich N, Becker-Santos DD, Pikor LA, Pusic A, Lockwood WW, Lam WL and Tsao MS: SOX15 is a candidate tumor suppressor in pancreatic cancer with a potential role in Wnt/ $\beta$-catenin signaling. Oncogene 33: 279-288, 2014.

32. Wang Y, Dang Y, Liu J and Ouyang X: The function of homeobox genes and lncRNAs in cancer. Oncol Lett 12: 1635-1641, 2016. 